\title{
Nonlinear Horizontal Diffusion for GCMs
}

\author{
ERICH BECKER \\ Leibniz-Institut für Atmosphärenphysik an der Universität Rostock e. V., Kühlungsborn, Germany \\ ULRIKE BURKHARDT \\ DLR-Institut für Physik der Atmosphäre, Oberpfaffenhofen, Wessling, Germany
}

(Manuscript received 6 October 2005, in final form 31 May 2006)

\begin{abstract}
The mixing-length-based parameterization of horizontal diffusion, which was originally proposed by Smagorinsky, is revisited. The complete tendencies of horizontal momentum diffusion, the associated frictional heating, and horizontal diffusion of sensible heat in spherical geometry are derived. The formulations are modified for the terrain-following vertical-hybrid-coordinate system in a way that ensures energy and angular momentum conservation at each layer. Test simulations with a simple general circulation model, run at T42 horizontal resolution and for permanent January conditions, confirm the conservation properties and highlight the enhancement of nonlinear horizontal diffusion in areas of high baroclinic activity. The simulated internal variability is dependent on the nature of the horizontal diffusion, with high-frequency variability being enhanced over the northern continents and low-frequency variability being increased (decreased) over the Pacific (Atlantic) Ocean when using nonlinear rather than linear diffusion. Locally reduced horizontal dissipation over Europe is compensated by increased dissipation owing to vertical diffusion, indicating the potential importance of nonlinear horizontal diffusion for gravity waveresolving simulations. Inspection of the spectral energy reveals that the scheme needs to be modified in order to damp unbalanced ageostrophic motions at the smallest resolved scales more efficiently. A corresponding empirical modification is proposed and proves to work properly.
\end{abstract}

\section{Introduction}

Atmospheric circulation models that resolve synoptic-scale waves need to contain horizontal diffusion in order to prevent the accumulation of enstrophy and kinetic energy at the smallest resolved scales. Horizontal diffusion (other equivalent terms are lateral or scaleselective horizontal damping), therefore, offsets the enstrophy cascade in the free atmosphere and helps to parameterize the dissipation of kinetic energy at unresolved scales (Tung and Orlando 2003). In the climatological mean, the adiabatic conversion of available potential energy into kinetic energy must be balanced by the irreversible loss of kinetic energy due to friction. In a model this loss is, in addition to boundary layer friction, to a significant extent due to horizontal momen-

Corresponding author address: Erich Becker, Leibniz-Institut für Atmosphärenphysik an der Universität Rostock e.V., Schlossstraße 6, 18225 Kühlungsborn, Germany.

E-mail: becker@iap-kborn.de tum diffusion (Becker 2003a). Therefore, the efficiency of the Lorenz energy cycle and the kinetic energy spectra simulated by circulation models are highly sensitive to the order and strength of the horizontal diffusion scheme (e.g., Barnes and Young 1992; Alexeev et al. 1996; Smith 2004; Tung 2004). Moreover, enhanced horizontal diffusion is often used to impose a sponge layer, which atmospheric circulation models need in order to control wave reflection at the model top (DKRZ 1992; Roeckner et al. 1996).

In atmospheric circulation models lateral damping may be applied using numerical filters or may be implicitly included in advanced advection schemes (e.g., Thuburn 1997). Most commonly though, an explicit scale-selective damping is added to the equations of motion. Such an explicit method is either linear or nonlinear. In a linear scheme, the spectral damping coefficients are prescribed such that the resulting tendencies are linear in the horizontal wind and temperature fields. Alternatively, the diffusion coefficient can be defined as a nonlinear function of the horizontal wind,

DOI: 10.1175/MWR3348.1

(C) 2007 American Meteorological Society 
and in this case the horizontal diffusion is said to be nonlinear.

Linear schemes are usually applied in spectral models, because they can easily be computed in spectral space. To achieve a high-scale selectivity, the diffusion is raised to biharmonic or higher orders (e.g., Boville 1991; Kiehl et al. 1996; Roeckner et al. 1996) or the damping coefficients for the spectral amplitudes are chosen empirically (e.g., Laursen and Eliasen 1989; DKRZ 1992). However, when using hyperdiffusion, angular momentum conservation is usually violated and a physically consistent dissipative heating cannot be formulated (Becker 2001, 2003b), the inclusion of which has been shown to cause statistically significant changes in the model climate (Burkhardt and Becker 2006).

Nonlinear horizontal diffusion was originally proposed by Smagorinsky (1963). Assuming a Cartesian coordinate system, he derived a nonlinear coefficient for horizontally isotropic diffusion, which is proportional to the norm of the rate of strain dyadic (hereafter: strain tensor). This method might also be interpreted as a mixing-length concept when the ideas of Prandtl are applied to the dissipation of enstrophy in two-dimensional turbulence. In this respect, Smagorinsky's nonlinear parameterization is more physically based than any linear scheme. When using explicit diffusion, nonlinear schemes have therefore become the preferred method in gridpoint models, in which a linear scheme is not technically advantageous. Furthermore, using nonlinear harmonic diffusion enables the consistent formulation of momentum diffusion and dissipative heating while damping only at times and places of strong horizontal shear.

Various modifications of Smagorinsky's formulation from 1963 have been proposed and implemented in gridpoint models developed since then. The original horizontal diffusion coefficient (Smagorinsky 1963) yields

$$
K_{h} \propto \sqrt{\left(\partial_{x} u-\partial_{y} v\right)^{2}+\left(\partial_{x} v+\partial_{y} u\right)^{2}} .
$$

Here, $u$ and $v$ denote the zonal and meridional wind and $\partial_{x}=\left(a_{e} \cos \phi\right)^{-1} \partial_{\lambda}$ and $\partial_{y}=a_{e}^{-1} \partial_{\phi}$, where $a_{e}$ is the earth's radius, $\lambda$ is longitude, and $\phi$ is latitude. A straightforward implementation of this diffusion coefficient would be problematic when using spherical geometry and would result in pole problems. Therefore, Eq. (1) needs to be modified. For instance, Andrews et al. (1983) introduced the form

$$
K_{h} \propto \sqrt{\left(\left|\partial_{x} u\right|+\left|\partial_{y} v\right|\right)^{2}+\left(\left|\partial_{x} v\right|-\left|\partial_{y} u\right|\right)^{2}},
$$

which is still in use in the SKYHI middle-atmosphere general circulation model (K. Hamilton 2003, personal communication). Grell et al. (1995) and Alexeev et al. (1996) used a nonlinear diffusion coefficient proportional to Eq. (1) along with a biharmonic diffusion of zonal and meridional wind components. Another modification of Smagorinsky's original formula was suggested by Gordon and Stern (1982). Their diffusion coefficient may be written as [see Eqs. (49)-(56) in their paper]

$$
K_{h} \propto \sqrt{\left(\partial_{x} u-v \tan \phi / a_{e}\right)^{2}+\left(\partial_{x} v+u \tan \phi / a_{e}\right)^{2}} .
$$

Later on, Smagorinsky (1993) himself proposed a modification. His revised method yields

$$
K_{h} \propto \sqrt{\begin{array}{l}
\left(\partial_{x} u-\partial_{y} v-v \tan \phi / a_{e}\right)^{2} \\
+\left(\partial_{x} v+\partial_{y} u+u \tan \phi / a_{e}\right)^{2}
\end{array}}
$$

and applies consistently in spherical geometry, as will be confirmed in the next section. As far as we know, this new horizontal diffusion coefficient has not been implemented in GCMs or mesoscale models yet, nor has the corresponding scheme been elaborated to such an extent as to provide the complete set of diffusion and dissipation tendencies for modelers.

The intention of the present study is to provide a complete recipe of how to incorporate the ideas of Smagorinsky (1993) in GCMs or mesoscale models that require spherical geometry. Particular emphasis is spent on the first principles of hydrodynamics and on conservation properties associated with the vertical discretization. The outline of the paper is as follows. In section 2 we adopt the stress-tensor formulation of Becker (2001, 2003a) and show how Smagorinsky's nonlinear horizontal diffusion coefficient can be interpreted as a generalization of Prandtl's mixing-length concept. Then we derive the expressions for friction, dissipation, and diffusion of sensible heat (temperature) with the geometric height $z$ as the vertical coordinate. Section 3 provides modifications such that the scheme conserves angular momentum and energy in any vertical-hybrid-coordinate system. In section 4 , the new formulations are tested using an idealized spectral troposphere-stratosphere GCM. On the one hand, life cycle experiments are performed in order to study the conservation properties of the nonlinear horizontal diffusion scheme (section 4a). On the other hand, climate runs are analyzed to study the changes in the diffusion, dissipation, and large-scale variability patterns when replacing linear by nonlinear horizontal diffusion (section $4 \mathrm{~b})$. We furthermore propose an extension of Smagorinsky's idea in order to constrain the excitation of non- 
balanced ageostrophic motions at high horizontal wavenumbers. Our conclusions are given in section 5.

\section{General formulation}

The two forms of the stress tensor that describe horizontally isotropic diffusion of horizontal momentum yield in spherical geometry (Becker 2001, 2003a,b):

$$
\begin{aligned}
\sum_{h}= & \rho K_{h}\left\{\left[\left(\boldsymbol{\nabla}+\mathbf{e}_{z} / a_{e}\right) \circ \mathbf{v}\right]+\left[\left(\boldsymbol{\nabla}+\mathbf{e}_{z} / a_{e}\right) \circ \mathbf{v}\right]^{\mathrm{T}}\right\} \text { and } \\
\sum_{h 0}= & \rho K_{h 0}\left\{\left[\left(\boldsymbol{\nabla}+\mathbf{e}_{z} / a_{e}\right) \circ \mathbf{v}\right]+\left[\left(\boldsymbol{\nabla}+\mathbf{e}_{z} / a_{e}\right) \circ \mathbf{v}\right]^{\mathrm{T}}\right. \\
& \left.-D\left(\mathbf{e}_{x} \circ \mathbf{e}_{x}+\mathbf{e}_{y} \circ \mathbf{e}_{y}\right)\right\} .
\end{aligned}
$$

Here, $\rho$ is density, and $\mathbf{v}=u \mathbf{e}_{x}+v \mathbf{e}_{y}$ and $\boldsymbol{\nabla}$ denote the horizontal velocity field and gradient operator, respectively. The unit vectors in zonal, meridional, and vertical direction are abbreviated as $\mathbf{e}_{x}, \mathbf{e}_{y}$, and $\mathbf{e}_{z}$, respectively, and the open circle denotes the tensor product. $^{1}$ Furthermore, the exponent $\mathrm{T}$ denotes the transpose, and $D=\boldsymbol{\nabla} \cdot \mathbf{v}=\partial_{x} u+\partial_{y} v-v \tan \phi / a_{e}$ is horizontal divergence. The tensor

$$
\begin{aligned}
\mathbf{S}_{h}= & {\left[\left(\boldsymbol{\nabla}+\mathbf{e}_{z} / a_{e}\right) \circ \mathbf{v}\right]+\left[\left(\boldsymbol{\nabla}+\mathbf{e}_{z} / a_{e}\right) \circ \mathbf{v}\right]^{\mathrm{T}} } \\
= & 2 \mathbf{e}_{x} \circ \mathbf{e}_{x}\left(D-\partial_{y} v\right)+\mathbf{e}_{x} \circ \mathbf{e}_{y}\left(\xi+2 \partial_{y} u\right) \\
& +\mathbf{e}_{y} \circ \mathbf{e}_{x}\left(\xi+2 \partial_{y} u\right)+2 \mathbf{e}_{y} \circ \mathbf{e}_{y} \partial_{y} v
\end{aligned}
$$

corresponds to twice the shallow-atmosphere analog of the tensor of deformation velocities (Sommerfeld 1978, his section 1) when retaining only the horizontal shear of the horizontal motion. Here, $\xi=\mathbf{e}_{z} \cdot(\boldsymbol{\nabla} \times \mathbf{v})=$ $\partial_{x} v-\partial_{y} u+u \tan \phi / a_{e}$ is horizontal vorticity. The geometric terms $\left(\mathbf{e}_{z} / a_{e} \circ \mathbf{v}\right)+\left(\mathbf{e}_{z} / a_{e} \circ \mathbf{v}\right)^{\mathrm{T}}$ ensure that the frictional stress at horizontally aligned Eulerian sectional planes vanishes, which is a necessary condition that any reasonable stress tensor for horizontal momentum diffusion must fulfill. We refer to $\mathbf{S}_{h}$ and

$$
\begin{aligned}
\mathbf{S}_{h 0}= & \mathbf{S}_{h}-D\left(\mathbf{e}_{x} \circ \mathbf{e}_{x}+\mathbf{e}_{y} \circ \mathbf{e}_{y}\right) \\
= & \mathbf{e}_{x} \circ \mathbf{e}_{x}\left(D-2 \partial_{y} v\right)+\mathbf{e}_{x} \circ \mathbf{e}_{y}\left(\xi+2 \partial_{y} u\right) \\
& +\mathbf{e}_{y} \circ \mathbf{e}_{x}\left(\xi+2 \partial_{y} u\right)-\mathbf{e}_{y} \circ \mathbf{e}_{y}\left(D-2 \partial_{y} v\right)
\end{aligned}
$$

as strain tensors. The index 0 indicates that the trace of the strain tensor $\mathbf{S}_{h 0}$ is zero, while it is $2 D$ for $\mathbf{S}_{h}$.

The nonlinear forms of $K_{h}$ and $K_{h 0}$ can be defined analogously to Prandtl's theory for turbulence in

\footnotetext{
${ }^{1}$ The convention is such that $(\mathbf{a} \circ \mathbf{b}) \mathbf{c}=\mathbf{a}(\mathbf{b} \cdot \mathbf{c})$ and $\mathbf{a}(\mathbf{b} \circ \mathbf{c})=$ $(\mathbf{a} \cdot \mathbf{b}) \mathbf{c}$ for arbitrary vectors $\mathbf{a}, \mathbf{b}$, and $\mathbf{c}$.
}

boundary layer shear flow. Let us first recapitulate that the vertical momentum diffusion is described by the stress tensor

$$
\sum_{z}=\rho K_{z} \mathbf{S}_{z}
$$

with the corresponding strain tensor given by

$$
\mathbf{S}_{z}=\mathbf{e}_{z} \circ \partial_{z} \mathbf{v}+\partial_{z} \mathbf{v} \circ \mathbf{e}_{z} .
$$

The well-known mixing-length formulation of the vertical diffusion coefficient is

$$
K_{z}=l_{z}^{2} \sqrt{\left(\partial_{z} \mathbf{v}\right)^{2}+S_{z \min }^{2}}=l_{z}^{2} \sqrt{\left|\mathbf{S}_{z}\right|^{2}+S_{z \min }^{2}} .
$$

Here, $l_{z}$ is a mixing length and $\left|\mathbf{S}_{z}\right|=\sqrt{\left(\partial_{z} \mathbf{v}\right)^{2}}$ denotes the Frobenius norm of the strain tensor $\mathbf{S}_{z}$ divided by $\sqrt{2}$. Furthermore, a minimum wind shear $S_{z \min }$ is introduced such that spatial derivatives of $K_{z}$ are always defined. The frictional heating (dissipation) associated with vertical momentum diffusion can easily be expressed using the strain tensor norm

$$
\epsilon_{z}=K_{z}\left(\partial_{z} \mathbf{v}\right)^{2}=K_{z}\left|\mathbf{S}_{z}\right|^{2}
$$

The idea of Smagorinsky (1963) assumes that the relationship (11) between the turbulent diffusion coefficient and the corresponding strain tensor holds generally. For the horizontal diffusion coefficients in Eqs. (5) and (6) this leads to

$$
\begin{aligned}
K_{h} & =l_{h}^{2} \sqrt{\left|\mathbf{S}_{h}\right|^{2}+S_{h \min }^{2}} \text { and } \\
K_{h 0} & =l_{h}^{2} \sqrt{\left|\mathbf{S}_{h 0}\right|^{2}+S_{h \min }^{2},}
\end{aligned}
$$

where $l_{h}$ is a prescribed mixing length and $S_{h \min }$ is a minimum horizontal wind shear. Using Eqs. (7) and (8), the tensor norms can be shown to be

$$
\left|\mathbf{S}_{h}\right|=\left[2\left(D-\partial_{y} v\right)^{2}+2\left(\partial_{y} v\right)^{2}+\left(\xi+2 \partial_{y} u\right)^{2}\right]^{1 / 2} \text { and }
$$

$\left|\mathbf{S}_{h 0}\right|=\left[\left(D-2 \partial_{y} v\right)^{2}+\left(\xi+2 \partial_{y} u\right)^{2}\right]^{1 / 2}$.

Equations (14) and (16) are equivalent to Smagorinsky's formulation of the diffusion coefficient from 1993 [Eq. (4)]. Due to spherical geometry, it differs from the original diffusion coefficient in Smagorinsky [1963, his Eq. (4.24), see also Eq. (1) in the current paper] with respect to the $\tan \phi$ terms contained in $D$ and $\xi$. The alternative formulation [Eqs. (13) and (15)] is simply the nonlinear diffusion coefficient that corresponds to the stress-tensor formulation proposed in Becker (2001), which is, except for the trace of the strain tensor, equivalent to Smagorinsky's ansatz. 
We procede in deriving the complete friction and frictional heating tendencies associated with the strain tensor in Eq. (7) and the corresponding diffusion coefficient in Eq. (13). Making no scaling assumption for $\rho$ or $K_{h}$, the tendency of the horizontal wind vector owing to horizontal momentum diffusion must be written as

$$
\begin{aligned}
\mathbf{H} & =\rho^{-1} \boldsymbol{\nabla}\left(\rho K_{h} \mathbf{S}_{h}\right) \\
& =\underbrace{K_{h} \boldsymbol{\nabla} \mathbf{S}_{h}}_{=: \mathbf{H}_{1}}+\underbrace{\mathbf{S}_{h}\left(\boldsymbol{\nabla} K_{h}\right)}_{=: \mathbf{H}_{2}}+\underbrace{\rho^{-1} K_{h} \mathbf{S}_{h}(\boldsymbol{\nabla} \rho)}_{=: \mathbf{H}_{3}} .
\end{aligned}
$$

The first term on the rhs of Eq. (17) has been given in Becker (2001):

$$
\mathbf{H}_{1}=K_{h}\left(\nabla^{2} \mathbf{v}+\nabla D+2 \mathbf{v} / a_{e}^{2}\right) .
$$

In the nonlinear case, this formula must be evaluated in gridpoint space. In a spectral GCM, where vorticity $\xi$ and divergence $D$ are used as prognostic variables, it is advantageous to express $\nabla^{2} \mathbf{v}$ in terms of these variables. According to $\nabla^{2} \mathbf{v}=\mathbf{e}_{z} \times \nabla \xi+\nabla D$ we get

$$
\begin{aligned}
\mathbf{H}_{1}= & K_{h}\left[\mathbf{e}_{x}\left(-\partial_{y} \xi+2 \partial_{x} D+2 u / a_{e}^{2}\right)\right. \\
& \left.+\mathbf{e}_{y}\left(\partial_{x} \xi+2 \partial_{y} D+2 v / a_{e}^{2}\right)\right] .
\end{aligned}
$$

The explicit form of $\mathbf{H}_{2}$ is

$$
\begin{aligned}
\mathbf{H}_{2}= & \frac{l_{h}^{2}}{2} \frac{1}{\sqrt{\left|\mathbf{S}_{h}\right|^{2}+S_{h \min }^{2}}}\left\{\mathbf { e } _ { x } \left[2\left(D-\partial_{y} v\right) \partial_{x}\left|\mathbf{S}_{h}\right|^{2}\right.\right. \\
& \left.+\left(\xi+2 \partial_{y} u\right) \partial_{y}\left|\mathbf{S}_{h}\right|^{2}\right]+\mathbf{e}_{y}\left[2 \partial_{y} v \partial_{y}\left|\mathbf{S}_{h}\right|^{2}\right. \\
& \left.\left.+\left(\xi+2 \partial_{y} u\right) \partial_{x}\left|\mathbf{S}_{h}\right|^{2}\right]\right\},
\end{aligned}
$$

where

$$
\begin{aligned}
\partial_{x}\left|\mathbf{S}_{h}\right|^{2}= & 4\left(D-\partial_{y} v\right)\left(\partial_{x} D-\partial_{x} \partial_{y} v\right)+4 \partial_{y} v \partial_{x} \partial_{y} v \\
& +2\left(\xi+2 \partial_{y} u\right)\left(\partial_{x} \xi+2 \partial_{x} \partial_{y} v\right) \text { and } \\
\partial_{y}\left|\mathbf{S}_{h}\right|^{2}= & 4\left(D-\partial_{y} v\right)\left(\partial_{y} D-\partial_{y}^{2} v\right)+4 \partial_{y} v \partial_{y}^{2} v \\
& +2\left(\xi+2 \partial_{y} u\right)\left(\partial_{y} \xi+2 \partial_{y}^{2} u\right) .
\end{aligned}
$$

The friction terms $\mathbf{H}_{1}$ and $\mathbf{H}_{2}$ are principally of the same order of magnitude. Nevertheless, $\mathbf{H}_{2}$ is commonly ignored. The third term on the rhs of Eq. (17), $\mathbf{H}_{3}$, is negligible according to shallow atmosphere scaling. However, it might become important if we reformulate our horizontal diffusion scheme using a terrainfollowing vertical coordinate (see section 3 ). The frictional heating associated with $\mathbf{H}$ can be written analogously to Eq. (12):

$$
\epsilon_{h}=K_{h}\left(\mathbf{S}_{h} \boldsymbol{\nabla}\right) \cdot \mathbf{v}=K_{h}\left|\mathbf{S}_{h}\right|^{2} .
$$

Expressions very similar to Eqs. (17)-(23) are obtained when we adopt the zero-trace form of the strain tensor:

$$
\begin{aligned}
& \mathbf{H}_{0}=\underbrace{K_{h 0} \boldsymbol{\nabla} \mathbf{S}_{h 0}}_{=: \mathbf{H}_{10}}+\underbrace{\mathbf{S}_{h 0}\left(\boldsymbol{\nabla} K_{h 0}\right)}_{=: \mathbf{H}_{20}}+\underbrace{\rho^{-1} K_{h 0} \mathbf{S}_{h 0}(\boldsymbol{\nabla} \rho)}_{=: \mathbf{H}_{30}}, \\
& \mathbf{H}_{10}=K_{h 0}\left(\nabla^{2} \mathbf{v}+2 \mathbf{v} / a_{e}^{2}\right) \\
& =K_{h 0}\left[\mathbf{e}_{x}\left(-\partial_{y} \xi+\partial_{x} D+2 u / a_{e}^{2}\right)\right. \\
& \left.+\mathbf{e}_{y}\left(\partial_{x} \xi+\partial_{y} D+2 v / a_{e}^{2}\right)\right], \\
& \mathbf{H}_{20}=\frac{l_{h}^{2}}{2} \frac{1}{\sqrt{\left|\mathbf{S}_{h 0}\right|^{2}+S_{h \min }^{2}}}\left\{\mathbf { e } _ { x } \left[\left(D-2 \partial_{y} v\right) \partial_{x}\left|\mathbf{S}_{h 0}\right|^{2}\right.\right. \\
& \left.+\left(\xi+2 \partial_{y} u\right) \partial_{y}\left|\mathbf{S}_{h 0}\right|^{2}\right] \\
& +\mathbf{e}_{y}\left[-\left(D-2 \partial_{y} v\right) \partial_{x}\left|\mathbf{S}_{h 0}\right|^{2}\right. \\
& \left.\left.+\left(\xi+2 \partial_{y} u\right) \partial_{x}\left|\mathbf{S}_{h 0}\right|^{2}\right]\right\}, \\
& \partial_{x}\left|\mathbf{S}_{h 0}\right|^{2}=2\left(D-2 \partial_{y} v\right)\left(\partial_{x} D-2 \partial_{x} \partial_{y} v\right) \\
& +2\left(\xi+2 \partial_{y} u\right)\left(\partial_{x} \xi+2 \partial_{x} \partial_{y} u\right), \\
& \partial_{y}\left|\mathbf{S}_{h 0}\right|^{2}=2\left(D-2 \partial_{y} v\right)\left(\partial_{y} D-2 \partial_{y}^{2} v\right) \\
& +2\left(\xi+2 \partial_{y} u\right)\left(\partial_{y} \xi+2 \partial_{y}^{2} u\right), \quad \text { and } \\
& \epsilon_{h 0}=K_{h 0}\left(\mathbf{S}_{h \mathrm{o}} \boldsymbol{\nabla}\right) \cdot \mathbf{v}=K_{h 0}\left|\mathbf{S}_{h 0}\right|^{2} \text {. }
\end{aligned}
$$

When expressing the conventional momentum diffusion [Eq. (18)] in spectral space, we see that horizontal divergence is damped about twice as strong as horizontal vorticity (Becker 2001, 2003b). For the corresponding zero-trace formulation [Eq. (25)], vorticity and divergence have the same damping constants. This method is therefore expected to support the development of internal gravity waves and may be preferred in high-resolution simulations intended to explicitly describe gravity waves (Hamilton et al. 1995, 1999; Koshyk and Hamilton 2001; Becker and Fritts 2006). The former method, on the other hand, improves the consistency in low-resolution climate runs (Becker 2003a), where gravity waves need to be suppressed because their effects cannot adequately be resolved.

For harmonic diffusion, the horizontal diffusive flux of sensible heat is represented, as usual, by

$$
\mathbf{J}_{h}=-c_{p} P_{r}^{-1} K_{h} \rho \nabla T,
$$

where $c_{p}$ is the heat capacity at constant pressure and $P_{r}$ is the Prandtl number that is used to scale the heat diffusion relative to the momentum diffusion. Note that the exact evaluation of the horizontal convergence of Eq. (30) leads to additional terms just as in the case of momentum diffusion:

$$
\begin{aligned}
q_{h}= & -\rho^{-1} \boldsymbol{\nabla} \cdot \mathbf{J}_{h}=\underbrace{c_{p} P_{r}^{-1} K_{h} \nabla^{2} T}_{=: q_{1}}+\underbrace{c_{P} P_{r}^{-1} \boldsymbol{\nabla} T \cdot \nabla K_{h}}_{=: q_{2}} \\
& +\underbrace{c_{p} P_{r}^{-1} \rho^{-1} K_{h} \boldsymbol{\nabla} T \cdot \nabla \rho}_{=: q_{3}} .
\end{aligned}
$$


Again, the term that invokes $\nabla K_{h}$ (i.e., $q_{2}$ ) is usually ignored in GCMs with nonlinear horizontal diffusion, and the last term on the rhs of Eq. (31), $q_{3}$, may become important in other coordinate systems. If $K_{h 0}$ is used instead of $K_{h}$, the corresponding definitions for $\mathbf{J}_{h 0}, q_{h 0}$, $q_{10}, q_{20}$, and $q_{30}$ are analogous to Eqs. (30) and (31).

\section{Representation in a vertical-hybrid-coordinate system}

The method of Simmons and Burridge (1981) to use a terrain-following vertical coordinate that approaches pressure surfaces in the upper troposphere and farther above has become a standard method in both global and regional atmospheric circulation models. The question arises of how to modify the horizontal diffusion scheme described in the previous section for use with such a vertical-coordinate system in order to maintain its consistency with first principles of hydrodynamics. In Becker (2003a,b) an approximate method was proposed. The idea was that the scheme derived in the $z$ system can directly be applied in the hybrid-coordinate system, provided that the horizontal diffusion coefficient is set equal to zero in the lower troposphere, where hybrid surfaces can deviate substantially from height surfaces. The linear horizontal diffusion scheme was then to a good approximation angular momentum and energy conserving, at least on the global scale. In the present paper we propose a more rigorous method that ensures accurate energy and angular momentum conservation properties for global control volumes of finite vertical thickness in the hybrid-coordinate system.

For convenience, let us first consider horizontal diffusion of sensible heat, which in the $z$-coordinate system is defined by (31), or more precisely by

$$
q_{h}=c_{p} P_{r}^{-1} \rho^{-1} \nabla_{z} \cdot\left(K_{h} \rho \nabla_{z} T\right) .
$$

Here, the index $z$ denotes that the horizontal derivatives have to be evaluated at surfaces of constant height $z$. It is easily seen that

$a_{e}^{2} \int_{0}^{2 \pi} d \lambda \int_{-1}^{1} d \sin \phi \int_{z}^{z+\Delta z} d z \rho q_{h}=: \int_{z}^{z+\Delta z} d z \int d f \rho q_{h}=0$

with $d f$ describing the surface element spanned by $d \lambda$ and $d \sin \phi$. Equation (33) states that horizontal diffusion of sensible heat does not change the total potential energy of any atmospheric layer that has horizontally uniform boundaries with respect to height $z$ and is not intersected by topography. It is clear that a correspond- ing constraint must apply when we specify horizontal diffusion of heat in any other vertical-coordinate system. Here we choose the hybrid coordinate $\eta$ introduced by Simmons and Burridge (1981) with

$$
p(\lambda, \phi, \eta ; t)=a(\eta)+p_{s}(\lambda, \phi, t) b(\eta)
$$

and

$$
\eta=0 \text { for } p=0 \text { and } \eta=1 \text { for } p=p_{s},
$$

where $p$ is pressure and $p_{s}$ surface pressure. The functions $a(\eta)$ and $b(\eta)$ are prescribed. On the lowest half level, $a=0$ and $b=1$ so that the level follows exactly the topography. The coefficient $b$ monotonically decreases with increasing height whereas $a$ attains a maximum in the midtroposphere. In the middle atmosphere we have $a \gg b p_{s}$, which means that model layers correspond approximately to pressure levels. Invoking hydrostatic balance, the finite-difference analog of Eq. (33) yields in the hybrid-coordinate system:

$$
g^{-1} \int d f \Delta p_{l} q_{h l}=0 .
$$

Here, the index $l$ enumerates full model layers, running from $l=1$ for the uppermost layer to $l=1$ ev for the lowermost one. The associated pressure increments are given by

$$
\Delta p_{l}=\Delta a_{l}+p_{s}(\lambda, \phi, t) \Delta b_{l}>0,
$$

with $\Delta a_{l}=a_{l+1 / 2}-a_{l-1 / 2}$ and $\Delta b_{l}=b_{l+1 / 2}-b_{l-1 / 2}$ where half indices indicate intermediate half levels. The energy-conservation constraint [Eq. (36)] is satisfied if we define horizontal diffusion of heat at full model layers as

$$
q_{h l}=c_{p} P_{r}^{-1}\left(\Delta p_{l}\right)^{-1} \nabla_{\eta} \cdot\left(\Delta p_{l} K_{h l} \nabla_{\eta} T_{l}\right),
$$

where $\nabla_{\eta}$ denotes the horizontal gradient operator at surfaces of constant hybrid coordinate. In other words, we have substituted

$$
c_{p} P_{r}^{-1} \Delta z \rho K_{h} \nabla_{z} T \rightarrow c_{p} P_{r}^{-1} \frac{\Delta p_{l}}{g} K_{h l} \nabla_{\eta} T_{l}
$$

for the diffusive horizontal flux density of sensible heat multiplied by the height increment $\Delta z$. Accordingly, the following variables are redefined:

$$
\begin{aligned}
\mathbf{S}_{h l}= & {\left[\left(\boldsymbol{\nabla}_{\eta}+\mathbf{e}_{z} / a_{e}\right) \circ \mathbf{v}_{l}\right]+(\ldots)^{\mathrm{T}}, } \\
\left|\mathbf{S}_{h l}\right|= & {\left[2\left(D_{l}-\partial_{y} v_{l}\right)^{2}+2\left(\partial_{y} v_{l}\right)^{2}\right.} \\
& \left.+\left(\xi_{l}+2 \partial_{y} u_{l}\right)^{2}\right]^{1 / 2}, \text { and } \\
K_{h l}= & l_{h l}^{2}\left|\mathbf{S}_{h l}\right|,
\end{aligned}
$$


where all derivatives of the horizontal velocity field, including $\xi_{l}=\mathbf{e}_{z} \cdot\left(\boldsymbol{\nabla}_{\eta} \times \mathbf{v}_{l}\right)$ and $D_{l}=\boldsymbol{\nabla}_{\eta} \cdot \mathbf{v}_{l}$, are computed along surfaces of constant $\eta$.

The modifications of momentum diffusion and dissipation tendencies in the hybrid-coordinate system are

$$
\begin{aligned}
& \mathbf{H}_{l}=\left(\Delta p_{l}\right)^{-1} \nabla_{\eta}\left(\Delta p_{l} K_{h l} \mathbf{S}_{h l}\right) \quad \text { and } \\
& \epsilon_{h l}=K_{h l}\left(\mathbf{S}_{h l} \boldsymbol{\nabla}_{\eta}\right) \cdot \mathbf{v}_{l}=K_{h l}\left|\mathbf{S}_{h l}\right|^{2} .
\end{aligned}
$$

To prove energy conservation we consider the rate of change of kinetic plus thermal energy that is generated by horizontal momentum diffusion and dissipation at a full model layer and integrate by parts:

$$
\begin{aligned}
& g^{-1} \int d f \Delta p_{l}\left(\mathbf{v}_{l} \cdot H_{l}+\epsilon_{h l}\right) \\
& =g^{-1} \int d f\left\{\mathbf{v}_{l} \cdot\left[\nabla_{\eta}\left(\Delta p_{l} K_{h l} \mathbf{S}_{h l}\right)\right]+\left(\Delta p_{l} K_{h l} \mathbf{S}_{h l} \boldsymbol{\nabla}_{\eta}\right) \cdot \mathbf{v}_{l}\right\} \\
& =g^{-1} \int d f \boldsymbol{\nabla}_{\eta} \cdot\left(\Delta p_{l} K_{h l} \mathbf{S}_{h l} \mathbf{v}_{l}\right)=0
\end{aligned}
$$

Likewise, the rate of change of zonal angular momentum due to horizontal momentum diffusion yields for a full model layer:

$$
\begin{aligned}
g^{-1} \int d f a_{e} \cos \phi \mathbf{e}_{x} \cdot\left[\nabla_{\eta}\left(\Delta p_{l} K_{h l} \mathbf{S}_{h l}\right)\right] \\
=g^{-1} \int d f \boldsymbol{\nabla}_{\eta} \cdot\left(a_{e} \cos \phi \Delta p_{l} K_{h l} \mathbf{e}_{x} \mathbf{S}_{h l}\right) \\
\quad-g^{-1} \int d f\left(\Delta p_{l} K_{h l} \mathbf{S}_{h l} \boldsymbol{\nabla}_{\eta}\right) \cdot\left(a_{e} \cos \phi \mathbf{e}_{x}\right) .
\end{aligned}
$$

The first term on the rhs of Eq. (46) evidently vanishes. Due to the symmetry of $S_{h l}$ and the derivatives $\partial_{x} \mathbf{e}_{x}=$ $a_{e}^{-1}\left(\tan \phi \mathbf{e}_{y}-\mathbf{e}_{z}\right)$ and $\partial_{y} \mathbf{e}_{x}=0$ also the second term on the rhs of Eq. (46) is zero. Hence, angular momentum is not changed due to horizontal diffusion:

$$
\int d f a_{e} \cos \phi \mathbf{e}_{x} \cdot\left[\nabla_{\eta}\left(\Delta p_{l} K_{h l} \mathbf{S}_{h l}\right)\right]=0
$$

Equations (36), (45), and (47) hold equally for the stress-tensor formulation with zero trace.

Summarizing, the nonlinear horizontal diffusion scheme as defined in the previous section can be directly applied in a hybrid-vertical-coordinate system. We merely have to substitute all horizontal derivatives along surfaces of constant $z$ by the corresponding derivatives along surfaces of constant $\eta$ and, in addition,
$\mathbf{H}_{3}$ or $\mathbf{H}_{30}$ and $q_{3}$ or $q_{30}$ [see Eqs. (24) and (31)] must be substituted by the following expressions:

$$
\begin{aligned}
\mathbf{H}_{3 l} & =\frac{1}{\Delta p_{l}} K_{h l} \Delta b_{l} \mathbf{S}_{h l} \nabla_{\eta} p_{s}, \\
\mathbf{H}_{30 l} & =\frac{1}{\Delta p_{l}} K_{h 0 l} \Delta b_{l} \mathbf{S}_{h 0 l} \nabla_{\eta} p_{s}, \\
q_{3 l} & =\frac{c_{p} / P_{r}}{\Delta p_{l}} K_{h l} \Delta b_{l} \nabla_{\eta} T_{l} \cdot \nabla_{\eta} p_{s}, \quad \text { and } \\
q_{30 l} & =\frac{c_{p} / P_{r}}{\Delta p_{l}} K_{h 0 l} \Delta b_{l} \nabla_{\eta} T_{l} \cdot \nabla_{\eta} p_{s} .
\end{aligned}
$$

The modified forms of heat and momentum diffusion [Eqs. (38) and (43)] in the hybrid-coordinate system are similar to the diffusion tendencies proposed by Gordon and Stern [1982, their Eqs. (49) and (56)] for $\sigma$ coordinates. Note that our modified nonlinear diffusion scheme precisely conserves energy and angular momentum on the global scale only. Still it is questionable whether application of any horizontal diffusion is reasonable in regions of steep orography. Therefore, for climate simulations (section $4 \mathrm{~b}$ ) we set the horizontal mixing length $l_{h}(\eta)$ equal to zero in the lower troposphere. Only for the life cycle experiments (section 4a), where we do not prescribe any orography, we use a height-independent mixing length $l_{h}$.

\section{Numerical experiments with a simple GCM}

In this section we test the proposed formulation of nonlinear horizontal diffusion in a simple general circulation model, the Kühlungsborn Mechanistic General Circulation Model (KMCM), which has extensively been described in Becker and Schmitz (2001), Becker (2003b), and Körnich et al. (2003). KMCM has been modified so that all tendencies owing to horizontal diffusion and dissipation are computed in gridpoint space. All horizontal derivatives required by the diffusion scheme are obtained using the spectral transform method.

In the following we first inspect baroclinic life cycle experiments in order to validate energy and angularmomentum conservation independently from any other physical parameterization. Then we deal with the model climatology in perpetual January simulations and discuss the spatial distribution of the simulated horizontal and vertical diffusion coefficients and the associated frictional heating rates in the upper troposphere. This is done by using the nonlinear or the linear horizontal diffusion scheme and tuning the mixing 
length or the diffusion coefficient such as to reproduce the observed time-mean large-scale circulation.

\section{a. Life cycle experiments}

We follow Simmons and Hoskins (1978) and compute the temporal evolution of a baroclinic wave in a mechanically and thermally isolated model atmosphere, that is, in an atmosphere where horizontal diffusion is the only physical parameterization. Such a model atmosphere exchanges neither angular momentum nor energy with the surroundings. Becker (2001, 2003b) showed that linear harmonic horizontal diffusion formulated via the symmetric stress tensor [Eq. (5)] complies with these constraints. Here we ask to what extent the proposed nonlinear scheme is angular momentum and energy conserving as well.

In particular, we monitor relative and $\Omega$-angular momentum, associated with the earth rotation, global energies, and energy conversion rates due to diffusion and frictional heating for different setups of the horizontal diffusion scheme. Each simulation is carried out using T42 spectral resolution and 30 equally spaced hybrid levels. A globally uniform horizontal mixing length squared of $l_{h}^{2}=7 \times 10^{9} \mathrm{~m}^{2}$ is assumed along with $P_{r}=$ 5 and $S_{h \min }^{2}=10^{-10} \mathrm{~s}^{-2}$. The latter was chosen such as to minimize aliasing errors in the spectral transform method. The initial condition consists of a thermally balanced zonal flow that is perturbed by the most unstable normal mode of zonal wavenumber 6 .

Figure 1 shows results for the so-defined control simulation that includes nonlinear diffusion and the associated dissipative heating. The solid lines correspond to total kinetic energy (TKE) and available potential energy (APE; Lorenz 1955). To evaluate energy conservation, total energy [TKE + total potential energy (TPE)] and TPE are plotted as well (dashed lines). Note that these energies include offsets to fit into the diagrams.

During the phase of baroclinic instability, TKE grows at the cost of APE while TPE changes like APE. Even before TKE reaches a maximum, the energy transfer becomes irreversible. This is evident because unavailable potential energy (i.e., TPE - APE; Lorenz 1967), gradually increases as a consequence of frictional heating. The generation of unavailable potential energy is strongest around the life cycle maximum. At the end of the life cycle, the generated unavailable potential energy is of the same order of magnitude as the total kinetic energy contained in the model atmosphere. In particular, total energy is precisely conserved.

The situation is different when frictional heating is ignored. Figure 1 includes additional results from a con-
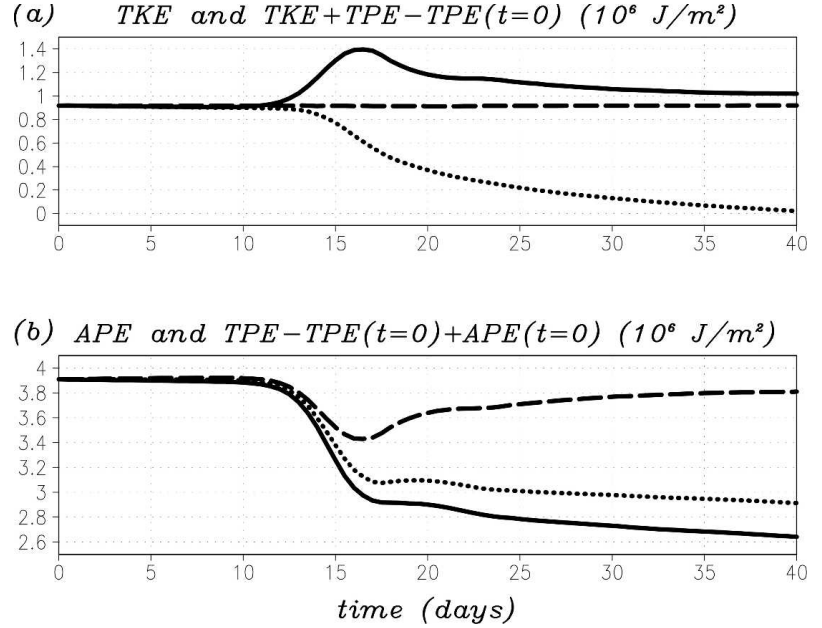

FIG. 1. Temporal evolution of global energies $\left(10^{6} \mathrm{~J} \mathrm{~m}^{-2}\right)$ in life cycle experiments with nonlinear horizontal diffusion. (a) TKE in the control run (solid line), and total energy defined as TKE + $\operatorname{TPE}-\operatorname{TPE}(t=0)$ in the control run (dashed line) and in the conventional run with $\epsilon_{h}$ ignored (dotted line). (b) APE in the control run (solid line), and $\operatorname{TPE}-\operatorname{TPE}(t=0)+\operatorname{APE}(t=0)$ in the control run (dashed line) and in the conventional run with $\epsilon_{h}$ ignored (dotted line).

ventional simulation where the model was integrated as in the control case but with $\epsilon_{h}$ set to zero. Because TKE and APE are approximately the same as in the control simulation, these quantities are not shown for the run without dissipation. Total energy and total potential energy, on the other hand, exhibit strong deviations from the control run (cf. dotted and dashed lines in Fig. 1). Because no unavailable potential energy is generated due to dissipation of kinetic energy, the temporal behavior of TPE is close to that of APE. In particular, total energy is not conserved anymore when $\epsilon_{h}$ is ignored.

For the control simulation (including dissipative heating) Fig. 2a shows the temporal evolution of relative angular momentum $L_{r}$ (solid line) together with total angular momentum (dashed line). The latter is defined as $L_{r}$ plus $\Omega$-angular momentum $L_{0}$ and is plotted with an offset of $-L_{0}(t=0)$. Compared with the changes of $L_{r}$, total angular momentum is well conserved in the life cycle experiment.

To estimate the importance of the diffusion terms that arise from $\boldsymbol{\nabla} K_{h}$ (i.e., $\mathbf{H}_{2}$ and $q_{2}$ ) or from surface pressure variations (i.e., $\mathbf{H}_{3}$ and $q_{3}$ ) we have performed two additional life cycle simulations where $\mathbf{H}_{2}+\mathbf{H}_{3}$ and $q_{2}+q_{3}$ or just $\mathbf{H}_{3}$ and $q_{3}$ have been set to zero (dotted line or thin solid line in Fig. 2b). It appears that the terms including the horizontal gradient of $K_{h}$ are very important for angular momentum conservation and for energy conservation (not shown) during the life cycle. 

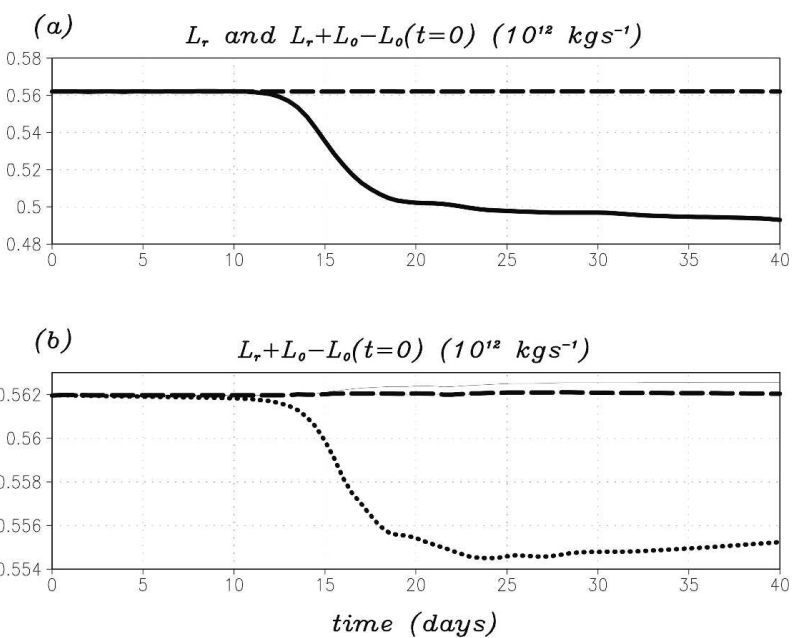

FIG. 2. (a) Temporal evolution of relative angular momentum (solid line; $10^{12} \mathrm{~kg} \mathrm{~s}^{-1}$ ) and total angular momentum (dashed line; $10^{12} \mathrm{~kg} \mathrm{~s}^{-1}$ ) in a life cycle experiment with the nonlinear horizontal diffusion scheme (control run). (b) Temporal evolution of total angular momentum in the control run [dashed line, same data as in (a)] and total angular momentum in two additional simulations where $\mathbf{H}_{2}+\mathbf{H}_{3}$ and $q_{2}+q_{3}$ (dotted line) or just the surface pressure corrections $\mathbf{H}_{3}$ and $q_{3}$ (thin solid line) are ignored.

The surface pressure corrections also improve the consistency of the present simulation, though their effect is rather weak since there is no orography.

\section{b. Perpetual January simulations}

In this section the response of the simulated climate to the introduction of a nonlinear instead of a linear horizontal diffusion coefficient is demonstrated. The distribution of horizontal and vertical diffusion and dissipation is analyzed and resulting changes in the internal variability patterns are shown.

\section{1) Model Setup}

For perpetual January simulations the model is modified in the following way. Orography is included. The hybrid levels are unequally spaced with enhanced resolution in the lower troposphere and in the stratosphere such that the uppermost full model layer is at $0.2 \mathrm{mb}$. Radiative heating is mimicked by temperature relaxation toward a zonally symmetric equilibrium temperature. Moist convective heating in the deep Tropics is represented by a prescribed additional heating, and latent heating in the midlatitudes is accounted for by selfinduced condensational heating. The equilibrium temperature and heating functions applied in the present model version differ slightly from those in previous versions of KMCM due to the different resolution used.
Boundary layer mixing is realized by using the local scheme described by Holtslag and Boville (1993) along with the energy-conserving vertical discretization of Becker (2003a). A prescribed vertical diffusion coefficient is added in the stratosphere with a maximum value of $10 \mathrm{~m}^{2} \mathrm{~s}^{-1}$ in the uppermost layer.

We have tuned the two diffusion schemes so that the model simulates the observed northern winter hemispheric circulation well [for an analysis of the climatology of KMCM see Becker and Schmitz (2001) and Körnich et al. (2003)]. In the nonlinear diffusion run, the horizontal mixing length squared is $l_{h}^{2}=5.2 \times 10^{8} \mathrm{~m}^{2}$ above $\eta=0.5$. Between $\eta=0.5$ and $0.75, l_{h}^{2}$ gradually decreases to zero (i.e., there is no horizontal diffusion below $\eta=0.75$ ). This slope has been introduced because horizontal diffusion on hybrid levels is not adequate in regions of steep orography and because the enstrophy cascade is generally not relevant in the lower troposphere. The other parameters are $S_{h \min }^{2}=0.4 \times$ $10^{-10} \mathrm{~s}^{-2}$ and $P_{r}=5$. The reflection of planetary waves at the model top is controlled by adding a linear horizontal diffusion coefficient in the stratosphere, which increases from zero to $2.3 \times 10^{6} \mathrm{~m}^{2} \mathrm{~s}^{-1}$ between $\eta=$ $0.06(60 \mathrm{hPa})$ and the top level at $\eta=0.002(0.2 \mathrm{hPa})$. In our linear diffusion run, the horizontal mixing length squared is zero everywhere and a linear diffusion coefficient of $K_{h}=6.5 \times 10^{4} \mathrm{~m}^{2} \mathrm{~s}^{-1}$ is used instead. This coefficient decays in the lower troposphere like $l_{h}^{2}$. The stratospheric sponge layer and all other model parameters are identical to those of the nonlinear diffusion run. Further simulations are defined in section $4 b(3)$.

Because the model is run at T42 horizontal resolution, baroclinic disturbances and their feedback on the large-scale flow are well resolved. After equilibration of the model climatologies, each setup has been integrated for another 2160 days. Model data have been sampled as snapshots every $12 \mathrm{~h}$. Differences between the two model simulations have been analyzed for significant changes due to the change in the horizontal diffusion scheme. Differences are assumed to be significantly larger than zero when they exceed a certain threshold associated with a $95 \%$ confidence interval according to the two-sided $t$-test statistic. The number of independent samples has been calculated accounting for serial dependence in the time series (Wilks 1995).

\section{2) Nonlinear versus Linear horizontal DIFFUSION: HORIZONTAL CROSS SECTIONS}

There exist a number of statistically significant differences between the two simulations. In the following we discuss changes in the internal variability and the efficiencies of horizontal and vertical dissipation. 
Figures $3 \mathrm{a}, \mathrm{b}$ show the climatological zonal wind at $300 \mathrm{hPa}$ for the nonlinear and the linear diffusion run. Both simulations reproduce the strength and location of the major northern winter hemispheric jets. Figures $3 \mathrm{c}$,d show the associated diffusion coefficients $K_{h}$ of the nonlinear and linear diffusion scheme at the $370-\mathrm{hPa}$ level, where the zonally averaged nonlinear $K_{h}$ is strongest (not shown) and maximizes in the areas of the storm tracks. In the climatological mean the uppertropospheric horizontal diffusion coefficient of the nonlinear scheme is between $50 \%$ and $80 \%$ smaller than the coefficient in the linear diffusion run. These differences are mainly because the constant diffusion coefficient in the linear diffusion run needs to be strong enough to damp the flow sufficiently at times and in regions of high baroclinic activity. The horizontal diffusion coefficient in the nonlinear diffusion run, on the other hand, is proportional to the horizontal shear and may be at times of high shear stronger but will usually be smaller than the constant coefficient in the linear diffusion run.

In both runs dissipative heating owing to horizontal momentum diffusion $\epsilon_{h}$ exhibits pronounced local maxima over the northeastern oceans. Figure 3e shows the resulting dissipation for the nonlinear diffusion run. This heating is nearly everywhere significantly smaller than in the linear diffusion run (Fig. 3f), except over the eastern Atlantic and western Europe, where changes do not exceed the statistical significance level of $95 \%$.

In the upper troposphere, the smaller $K_{h}$ and $\epsilon_{h}$ in the case of nonlinear horizontal diffusion are accompanied by a stronger vertical diffusion coefficient $K_{z}$ and dissipation $\epsilon_{z}$ (Fig. 4). (Note that the boundary layer parameterization with an asymptotic mixing length of $30 \mathrm{~m}$ for the vertical diffusion coefficient is also relevant in the upper troposphere.) In particular, both $K_{z}$ and $\epsilon_{z}$ are significantly enhanced in the regions of maximum internal variability over the eastern oceans. Apparently nonlinear diffusion allows for a stronger excitation of internal gravity waves, which are then damped by vertical diffusion since they have smaller vertical wave lengths than baroclinic waves. This interpretation is further supported by Fig. 5, which shows the transient kinetic energies at $300 \mathrm{hPa}$ associated with the divergent part of the flow, which may be regarded as a proxy for resolved gravity wave activity. This activity is clearly stronger in the nonlinear diffusion run, and the effect is most prominent over the eastern Atlantic.

Figure 6 illustrates the simulated transient kinetic energy patterns. In the nonlinear diffusion run the two elongated maxima of the transient kinetic energy, $\mathrm{KE}^{\prime}$, are captured (Fig. 6a), the latter extending from the central North Pacific to western Canada and from the western North Atlantic to western Europe (Roeckner et al. 1992, their Fig. A33a). Figure 6b reveals that the unfiltered $\mathrm{KE}^{\prime}$ is in the midlatitudes significantly stronger in the nonlinear diffusion run, except in the Atlantic area where $\mathrm{KE}^{\prime}$ is partly even reduced. The increase of $\mathrm{KE}^{\prime}$ over the continents is mainly due to a statistically significant increase in high-frequency variability (locally up to $20 \%$ ) with time scales of up to 10 days comprising mainly baroclinic disturbances (not shown). The low-frequency variability (with periods longer than 10 days) in the Pacific basin is significantly increased and shifted northward, whereas in the area of the Atlantic the low-frequency variability is decreased (not shown). Thus, we conclude that the patterns of internal variability depend on the choice of the diffusion scheme. Nonlinear diffusion supports a prolongation of the storm tracks over the continents and has regionally varying effects on the low-frequency variability.

\section{3) KinetiC ENERGy SPECTRA}

Kinetic energy spectra as defined by Koshyk et al. (1999) and averaged between 500 and $200 \mathrm{hPa}$ are shown in Fig. 7 for different model setups. In the nonlinear and linear diffusion run (black and gray solid lines in Fig. 7a), the $n^{-3}$ slope is prominent for synoptic scales between $n=10$ and 20. For $n<25$ the absolute energies compare well to those derived by Koshyk et al. (1999) from different comprehensive GCMs for January (see their Fig. 1a).

Both the nonlinear and the linear harmonic schemes are not capable of simulating the $n^{-3}$ slope at the highwavenumber end of the spectrum. We have done a number of tests, finding that this deficiency neither depends on the horizontal or vertical resolution nor on other model parameters or parameterizations. To further demonstrate this general shortcoming we have repeated the nonlinear run with a T85 horizontal resolution while all other model parameters, in particular the horizontal mixing length, are the same as in the corresponding T42 run. The T85 energy spectrum (dotted line in Fig. 7a) shows the $n^{-3}$ slope for $n$ between about 10 and 50. At smaller scales the spectrum flattens and increases for $n>75$. In this context it is worthwhile to note that the SKYHI model, which includes a nonlinear harmonic scheme according to Eq. (2), displays a similar behavior, depending on the procedure by which the spectrum is diagnosed (Koshyk and Hamilton 2001, see their Fig. 2).

The flattening of the energy spectrum for the highest wavenumbers suggests that harmonic diffusion is not capable of adequately parameterizing the simultaneous 
zonal wind at $300 \mathrm{mb}\left(\mathrm{ms}^{-1}\right)$

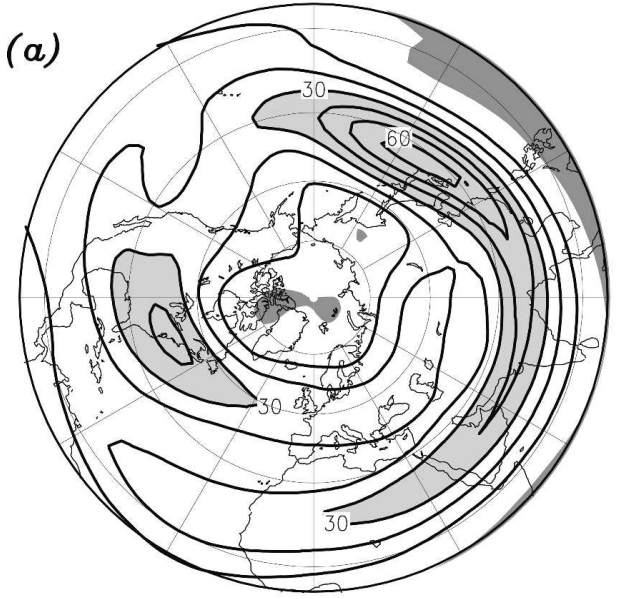

$K_{\mathrm{h}}\left(10^{3} \mathrm{~m}^{2} \mathrm{~s}^{-1}\right)$ at $370 \mathrm{mb}$

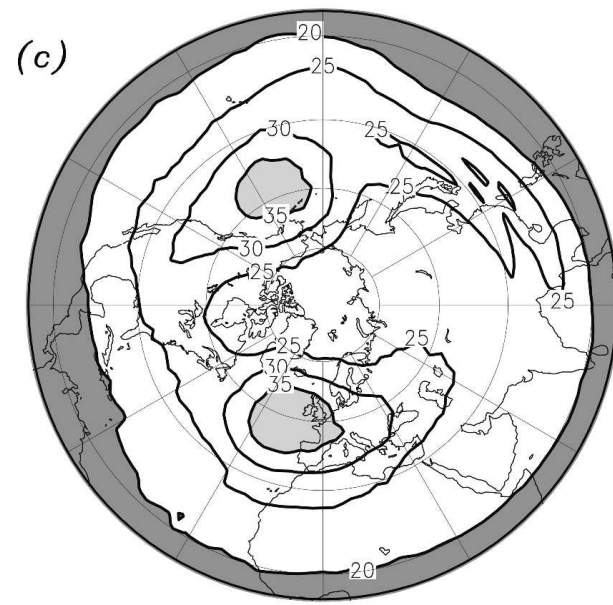

horiz. diss. $\left(10^{-3} \mathrm{Kd}^{-1}\right)$ at $370 \mathrm{mb}$

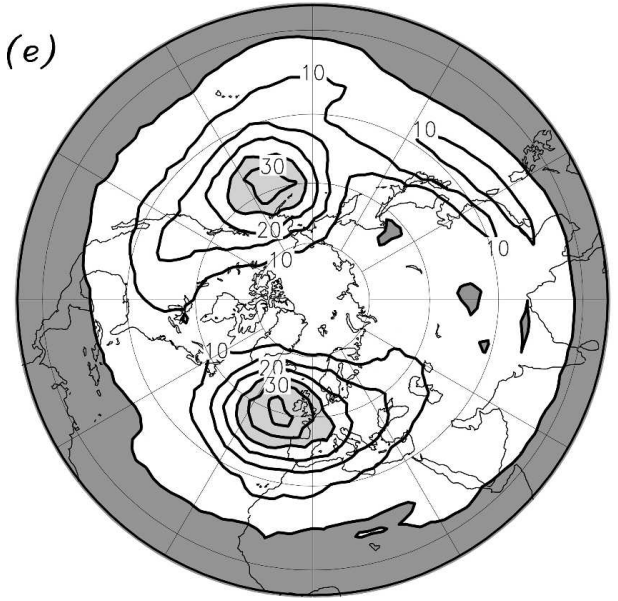

zonal wind at $300 \mathrm{mb}\left(\mathrm{ms}^{-1}\right)$

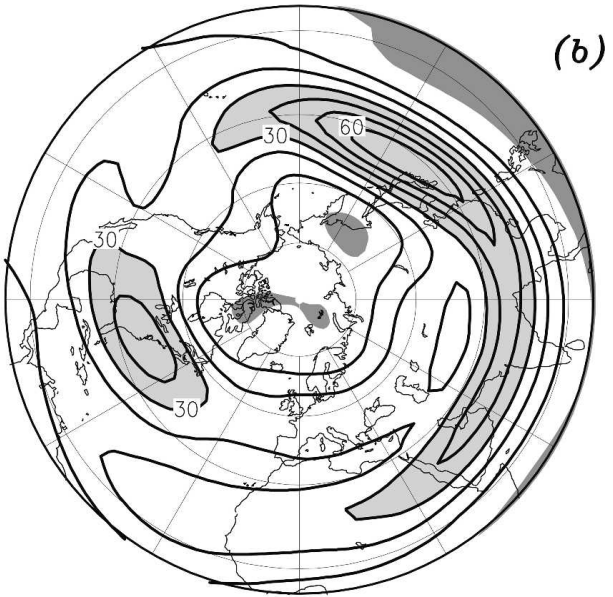

$K_{\mathrm{h}}\left(10^{3} \mathrm{~m}^{2} \mathrm{~s}^{-1}\right)$ at $370 \mathrm{mb}$

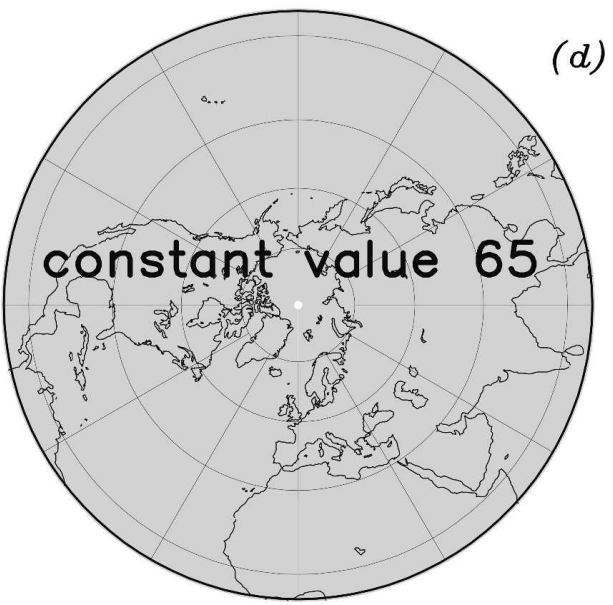

$\Delta$ horiz. diss. $\left(10^{-s} \mathrm{Kd}^{-1}\right)$ at $370 \mathrm{mb}$

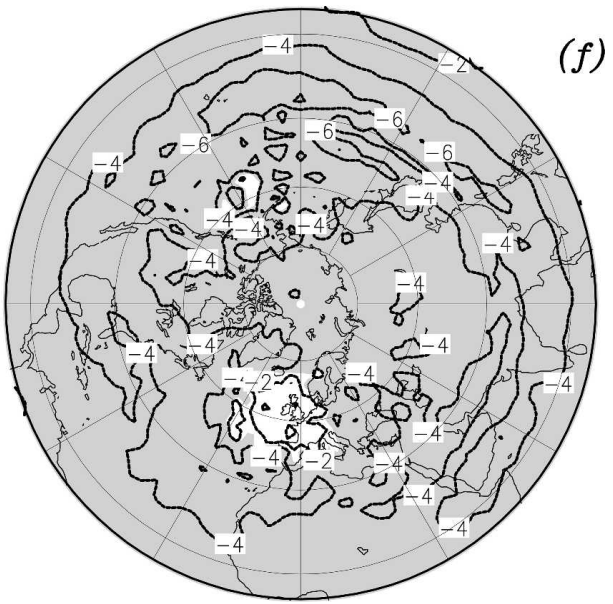

FIG. 3. Time-mean zonal wind at the hybrid level corresponding to $300 \mathrm{hPa}$ in the perpetual January simulations using (a) nonlinear and (b) linear horizontal diffusion. The contour interval is $10 \mathrm{~m} \mathrm{~s}^{-1}$. Easterlies are indicated by dark shading while light shading marks westerlies in excess of $30 \mathrm{~m} \mathrm{~s}^{-1}$. Time-mean horizontal diffusion coefficient at the hybrid level corresponding to $370 \mathrm{hPa}$ in the (c) 

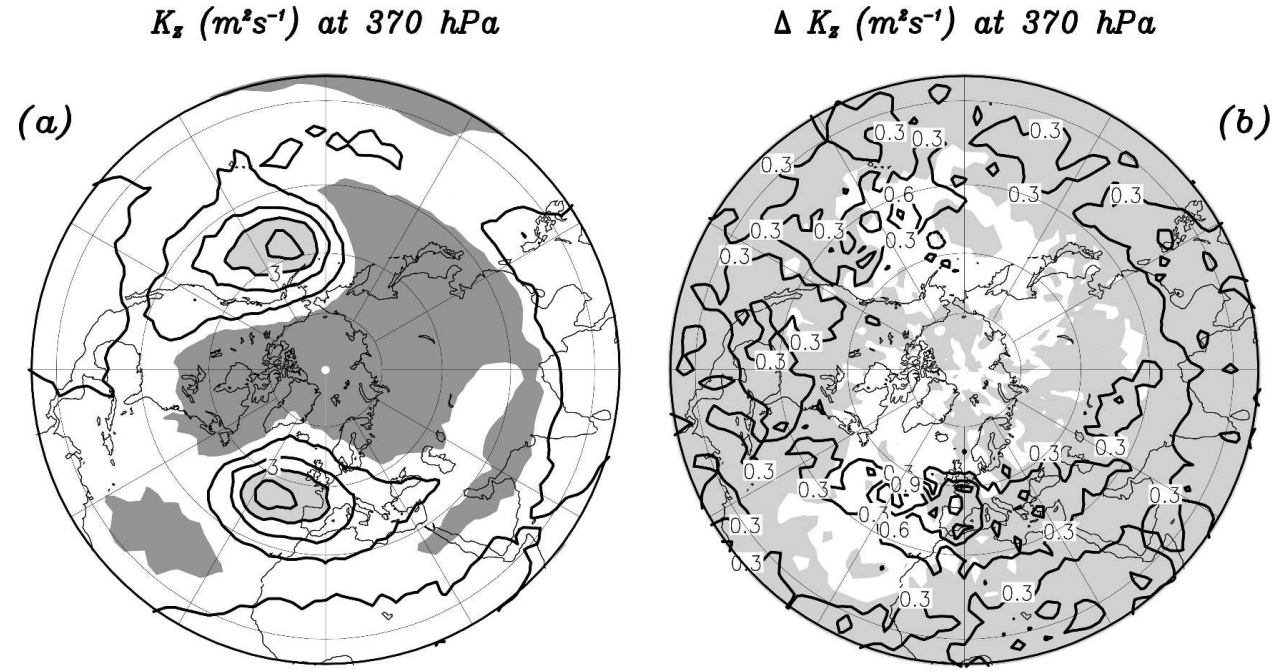

vert. diss. $\left(10^{-s} K^{-1}\right)$ at $370 \mathrm{hPa}$
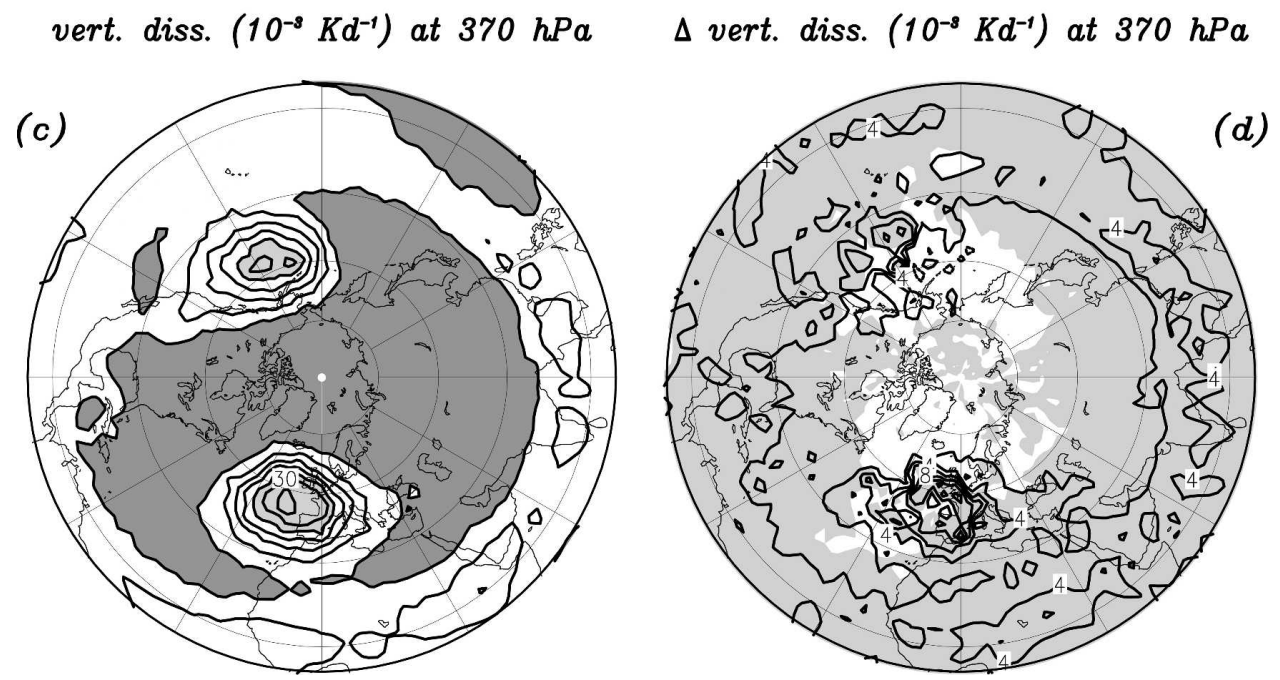

FIG. 4. (a) Time-mean vertical diffusion coefficient averaged between the two half hybrid levels adjacent to the $370-\mathrm{hPa}$ full level in the nonlinear diffusion run. The contour interval is $1 \mathrm{~m}^{2} \mathrm{~s}^{-1}$. Values less (greater) than $0.5(3) \mathrm{m}^{2} \mathrm{~s}^{-1}$ are indicated by dark (light) shading. (b) Same as in (a), but for the difference in the nonlinear from the linear diffusion run. The contour interval is $0.3 \mathrm{~m}^{2} \mathrm{~s}^{-1}$ with the zero contour omitted. Light shading indicates regions of statistical significance greater than $95 \%$. (c), (d) Same as in Figs. 3e,f, but for the dissipation owing to vertical momentum diffusion.

dissipation of enstrophy and energy at unresolved scales. On the other hand, a hyperdiffusion can easily be adjusted to yield the desired behavior in quasigeostrophic models (Tung and Orlando 2003), in a com- prehensive GCM (e.g., Boville 1991), and in the present model. In our hyperdiffusion run with KMCM (see the dotted line in Fig. 7b), $l_{h}^{2}$ was set to zero and instead the following spectral damping coefficients

$\leftarrow$

nonlinear and (d) linear diffusion run. The contour interval is $5 \times 10^{3} \mathrm{~m}^{2} \mathrm{~s}^{-1}$. Values less (greater) than $20 \times 10^{3}\left(35 \times 10^{-3}\right) \mathrm{m}^{2} \mathrm{~s}^{-1}$ are indicated by dark (light) shading. (e) Frictional heating due to horizontal momentum diffusion (horizontal dissipation) in the nonlinear diffusion run at the hybrid level corresponding to $370 \mathrm{hPa}$. The contour interval is $5 \times 10^{-3} \mathrm{~K}_{\text {day }}{ }^{-1}$. Values less (greater) than $5 \times 10^{-3}$ $\left(25 \times 10^{-3}\right) \mathrm{K} \mathrm{day}^{-1}$ are indicated by dark (light) shading. (f) Same as in (e), but for difference in the nonlinear from the linear diffusion run. The contour interval is $2 \times 10^{-3} \mathrm{~K}_{\text {day }}^{-1}$ with the zero contour omitted. Regions with statistical significance greater than $95 \%$ are indicated by light shading. 
div. $K E^{\prime}\left(m^{2} s^{-2}\right)$ at $300 \mathrm{hPa}$

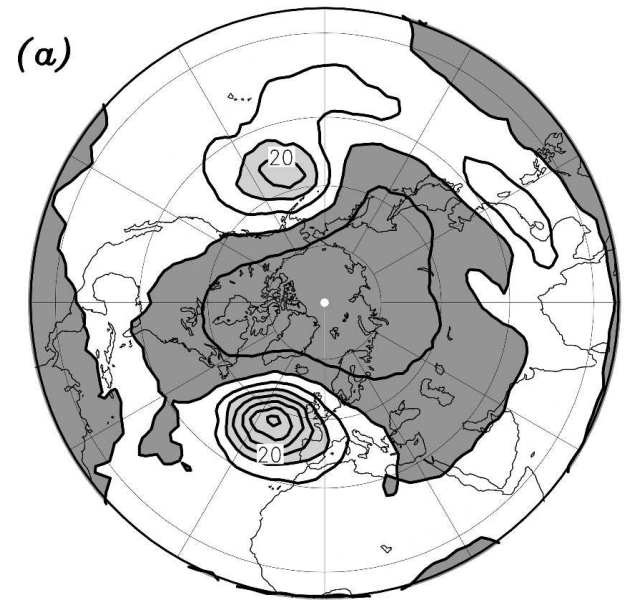

div. $\mathrm{KE}^{\prime}\left(\mathrm{m}^{2} \mathrm{~s}^{-2}\right)$ at $300 \mathrm{hPa}$

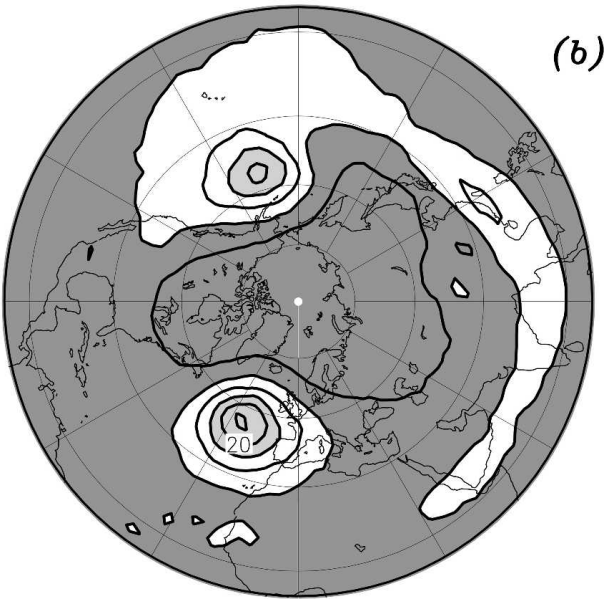

FIG. 5. Time-mean transient kinetic energy KE' owing to the nonrotational flow at $300 \mathrm{hPa}$ in (a) the nonlinear and (b) the linear diffusion run. The contour interval is $4 \mathrm{~m}^{2} \mathrm{~s}^{-2}$. Values less (greater) than 8 (16) $\mathrm{m}^{2} \mathrm{~s}^{-2}$ are indicated by dark (light) shading.

$\alpha_{n}=(0.6 \text { days })^{-1}\left\{\begin{array}{cc}(n-28)^{2} /(42-28)^{2} & \text { for } n>28 \\ 0 & \text { else }\end{array}\right.$

were applied to horizontal vorticity, as well as to horizontal divergence and temperature after multiplying by 2 and 0.2 , respectively. We also had to slightly retune the latent heating parameterization in order to main- tain a realistic simulation of the time-mean large-scale circulation.

In the scaling theory of two-dimensional turbulence (e.g., Salmon 1998, his chapter 4), the $n^{-3}$ spectrum can be derived when Prandtl's mixing-length assumption is applied to the dissipation of enstrophy. Smagorinsky's nonlinear harmonic diffusion should therefore be adequate to parameterize the dissipation of enstrophy at unresolved scales in atmospheric models. Nev- unfiltered $\mathrm{KE}^{\prime}\left(\mathrm{m}^{2} \mathrm{~s}^{-2}\right)$ at $300 \mathrm{hPa}$

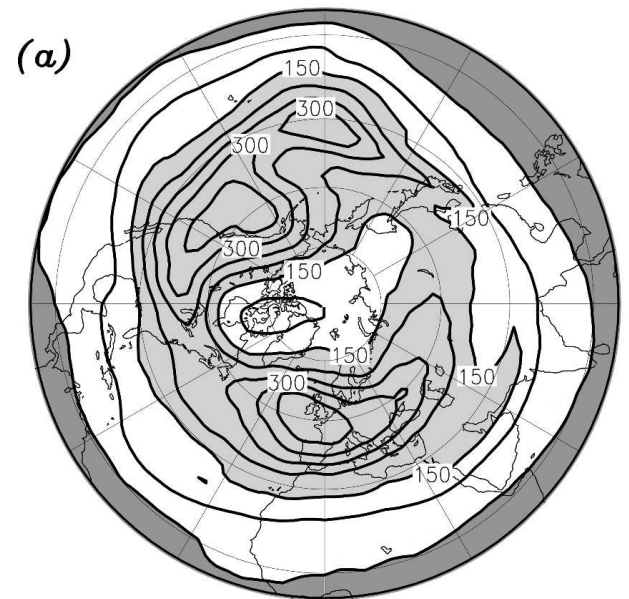

\section{$\Delta$ unfiltered $K E^{\prime}\left(m^{2} s^{-2}\right)$ at $300 h P a$}

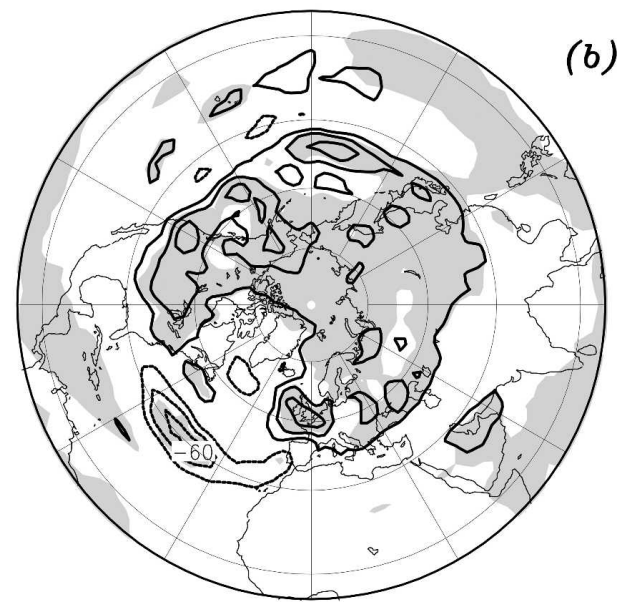

FIG. 6. (a) Unfiltered transient kinetic energy $\mathrm{KE}^{\prime}$ in the nonlinear diffusion run at the hybrid level corresponding to $300 \mathrm{hPa}$. The contour interval is $50 \mathrm{~m}^{2} \mathrm{~s}^{-2}$ and values less (greater) than $50(150) \mathrm{m}^{2} \mathrm{~s}^{-2}$ are indicated by dark (light) shading. (b) Same as in (a) but for the difference in the nonlinear from the linear diffusion run. The contour interval is $20 \mathrm{~m}^{2} \mathrm{~s}^{-2}$ and light shading indicates regions of statistical significance greater than $95 \%$. 

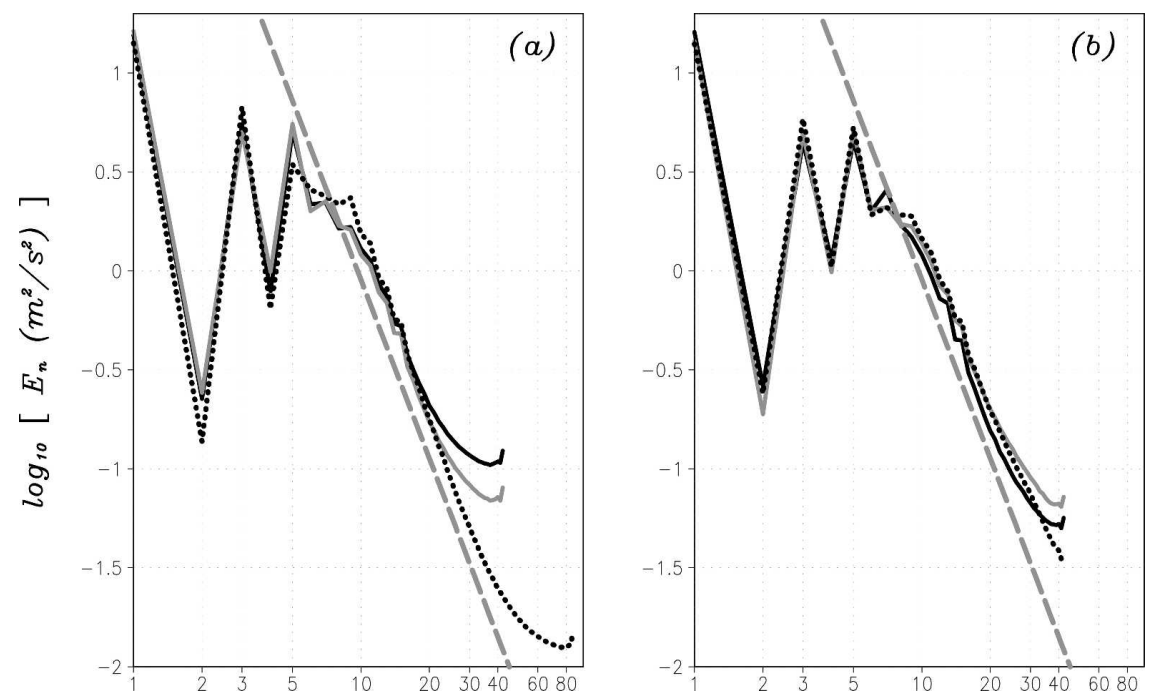

FIG. 7. Time-mean horizontal kinetic energy spectra averaged between 500 and $200 \mathrm{hPa}$ in perpetual January simulations. (a) In T42 resolutions using the nonlinear and the linear horizontal diffusion scheme (black and gray solid line, respectively), as well as in a T85 simulation using the nonlinear scheme (dotted line). (b) In T42 resolutions using the extended nonlinear scheme according to Eq. (54) (black solid line), using the nonlinear scheme supplemented by a hyperdiffusion of horizontal divergence according to Eq. (53) (gray solid line), and using a hyperdiffusion according to Eq. (52) instead of the nonlinear diffusion (dotted line). The dashed gray lines indicate the $n^{-3}$ slope.

ertheless, an application of the scheme can be problematic as a result of a net energy cascade, which accompanies the enstrophy cascade, and particularly due to unbalanced ageostrophic motions. These issues were extensively discussed by Tung and Orlando (2003), Smith (2004), and Tung (2004).

Unbalanced ageostrophic motions are mainly connected with gravity waves, for which the horizontal divergence $D$ is an adequate proxy (O'Sullivan and Dunkerton 1995). We therefore supplemented the nonlinear horizontal diffusion scheme by an additional hyperdiffusion of $D$, using the damping coefficients

$$
\beta_{n}=(0.1 \text { days })^{-1}\left\{\begin{array}{cc}
(n-23)^{2} /(42-23)^{2} & \text { for } n>23 \\
0 & \text { else. }
\end{array}\right.
$$

In this nonlinear hyperdiffusion run all other model parameters were kept up as in the hyperdiffusion run. The resulting energy spectrum deviates from the $n^{-3}$ slope only for $n>35$ (gray line in Fig. 7b). This result strongly suggests that the high-wavenumber ends of the spectra in Fig. 7a reflect an overestimation of unbalanced ageostrophic motions at the smallest resolved scales, rather than a deficiency of the Smagorinsky scheme to deal with quasigeostrophic flow. Accord- ingly, in order to yield an adequate energy spectrum Smagorinsky's scheme may be extended so that diffusion is enhanced when the horizontal divergence $D$ is strong. An empirical extension of Eq. (13) is

$$
K_{h}=l_{h}^{2}\left(1+\frac{D^{2}}{D_{\min }^{2}}\right) \sqrt{\left|\mathbf{S}_{h}\right|^{2}+S_{h \min }^{2}} .
$$

The corresponding modification of $K_{h 0}$, for which the trace of the strain tensor is zero [Eq. (14)], is analogous. The extended nonlinear diffusion coefficients require some straightforward changes of Eqs. (20) and (25) in order to maintain the conservation properties. In a correspondingly extended nonlinear diffusion run we have used Eq. (54) with $l_{h}=8.0 \mathrm{~km}$ and $D_{\min }=2 \times 10^{-6} \mathrm{~s}^{-1}$. All other parameters were again the same as in the hyperdiffusion run. The resulting energy spectrum (black line in Fig. 7b) is similar to the spectrum in the nonlinear hyperdiffusion run and it is even improved for the smallest scales. The hyperdiffusion run shows more energy at synoptic scales between about $n=10$ and 20 and less energy for $n>35$ (cf. the black solid and dotted lines in Fig. 7b). Therefore hyperdiffusion compared with our extended nonlinear diffusion has the advantage of being more scale selective, but at the expense of physical consistency. 
TABLE 1. Globally integrated time-mean frictional heating rates, $\left\langle\epsilon_{h}\right\rangle$ and $\left\langle\epsilon_{z}\right\rangle$, as well as the spurious thermal forcing $\Delta Q$ in the six different model simulations defined in sections $4 \mathrm{~b}(1)$ and $4 \mathrm{~b}(3)$. The weak $\left\langle\epsilon_{h}\right\rangle$ in the hyperdiffusion run, where in the troposphere $\epsilon_{h}=0$, is due to the linear harmonic diffusion in the stratospheric sponge layer.

\begin{tabular}{lcccr}
\hline \hline \multicolumn{1}{c}{ Lateral damping } & Horizontal resolution & $\left\langle\epsilon_{h}\right\rangle\left(\mathrm{W} \mathrm{m}^{-2}\right)$ & $\left\langle\epsilon_{z}\right\rangle\left(\mathrm{W} \mathrm{m}^{-2}\right)$ & $\Delta Q\left(\mathrm{~W} \mathrm{~m}{ }^{-2}\right)$ \\
\hline Nonlinear diffusion & $\mathrm{T} 42$ & 0.33 & 1.66 & 0.06 \\
Linear diffusion & $\mathrm{T} 42$ & 0.47 & 1.52 & -0.02 \\
Nonlinear diffusion & $\mathrm{T} 85$ & 0.48 & 1.73 & 0.03 \\
Extended nonlinear diffusion & $\mathrm{T} 42$ & 0.59 & 1.38 & -0.04 \\
Nonlinear hyperdiffusion & $\mathrm{T} 42$ & 0.21 & 1.54 & 0.19 \\
Hyperdiffusion & $\mathrm{T} 42$ & 0.03 & 1.61 & 0.38 \\
\hline
\end{tabular}

\section{4) GlOBAl BUdGETS}

An evaluation of the angular momentum and energy budgets for control volumes extending from the bottom to the model top and from the South Pole to some latitude farther north confirm the conservation properties of the diffusion-dissipation schemes (for details see Becker 2003a). These budgets compare the northward fluxes of relative angular momentum and total enthalpy with the integrals of the mountain and frictional torques and with the integrals of the diabatic heating rates due to differential heating, respectively (see also Lorenz 1967, his chapter IV). Similar to the experiments analyzed in Becker (2003a), the spurious global torque is negligible in all of our experiments, as it should be.

The spurious heating, defined as globally integrated and temporally averaged external diabatic heating (temperature relaxation, condensational heating, and sensible heating) should ideally vanish. However, because of parameterization errors, as well as numerical or aliasing errors, this is usually not the case in a global circulation model. The spurious heating should be compared to the approximate energy flux rate associated with the Lorenz energy cycle. According to Oort (1964), this rate is about $2 \mathrm{~W} \mathrm{~m}^{-2}$ for planetary and synoptic scales. As summarized in Table 1, the simulated spurious heating is about two orders of magnitude smaller than $2 \mathrm{~W} \mathrm{~m}^{-2}$ in all our experiments without any hyperdiffusion. Furthermore, the global integral of $\epsilon_{h}+\epsilon_{z}$ yields approximately that value in these runs. The spurious heating amounts to 0.19 or $0.38 \mathrm{~W} \mathrm{~m}^{-2}$ if horizontal diffusion in the upper troposphere is accompanied or substituted for by some hyperdiffusion (nonlinear hyperdiffusion and hyperdiffusion run).

\section{Conclusions}

The replacement of the linear by the nonlinear harmonic horizontal diffusion scheme allows for a consistent representation of momentum and thermodynamic tendencies while reducing the degree of damping of the large-scale flow. For this reason we have elaborated Smagorinsky's idea of a nonlinear horizontal diffusion coefficient (Smagorinsky 1963, 1993) by providing the complete set of wind tendencies [Eqs. (17), (19), (20), (24)-(26), (48), and (49)], as well as temperature tendencies owing to frictional heating [Eqs. (23) and (29)] and horizontal diffusion of sensible heat [Eqs. (30), (31), (50), and (51)]. Surface pressure corrections have been proposed that ensure precise global conservation properties on hybrid surfaces in GCMs that adopt the vertical differencing method of Simmons and Burridge (1981).

The consistency of the new formulations has been tested in life cycle experiments. We have demonstrated that during a life cycle the loss of kinetic energy is compensated for by an increase in unavailable potential energy so that total energy is conserved. The increase in unavailable energy is mainly caused by dissipative heating. Therefore, energy is only conserved when accounting for this process (Fig. 1). To comply with the conservation laws, the wind tendency that results from applying the strain tensor to the gradient of the diffusion coefficient can by no means be neglected. Also the proposed pressure correction term has a notable influence, even though its effect is very small in a calculation without orography.

With a mechanistic GCM (KMCM), driven by differential heating and comprising a local boundary layer scheme, we have performed two perpetual January simulations using either the nonlinear horizontal diffusion scheme or the corresponding linear scheme. In both experiments, the tuning of the horizontal mixing length or the diffusion coefficient is such that the simulated time-mean large-scale circulation is as observed. The simulations show that the introduction of nonlinearity in the diffusion coefficient changes the distribution of dissipative heating or the variability patterns due to more spatially and temporally localized damping. The associated nonlinear horizontal diffusion coefficient and dissipative heating are largest in the storm track areas and are generally weaker than their linear 
counterparts (Fig. 3). In contrast to the weakened horizontal diffusion and dissipation when using nonlinear horizontal diffusion, the vertical diffusion coefficient and the frictional heating owing to vertical momentum diffusion are significantly enhanced in the extratropical upper troposphere (Fig. 4), indicating enhanced gravity wave activity (Fig. 5). Also the patterns of internal variability exhibit statistically significant differences due to changing the horizontal diffusion scheme (Fig. 6). Using the nonlinear formulation, transient eddy kinetic energy is increased in the midlatitudes except in the Atlantic area. This change in the variability pattern results from an increased high-frequency variability over the continents and enhanced (reduced) low-frequency variability over the Pacific (Atlantic).

The gravity wave-supporting property of nonlinear horizontal diffusion may be of importance in highresolution simulations intended to explicitly describe gravity wave effects in the middle atmosphere (e.g., Hamilton et al. 1995, 1999; Koshyk and Hamilton 2001; Becker and Fritts 2006). It should however be noted that the generation of gravity waves in the present lowresolution model version is overestimated, as is indicated by an insufficient damping of the small-scale end of the kinetic energy spectrum (Fig. 7). Even though a mixing-length-based horizontal diffusion parameterizes the dissipation of enstrophy in two-dimensional turbulence, it can result in a buildup of energy at the smallest spatial scales in the presence of unbalanced ageostrophic motions. This deficiency can at least partly be corrected for by an extension of Smagorinsky's scheme, which allows us to deal with unbalanced ageostrophic motion while still remaining consistent with first principles. Such a modification is analogous to extending Prandtl's boundary layer diffusion by a Richardson criterion (e.g., Holtslag and Boville 1993). In the present paper we have tested an ad hoc method to provide an extended nonlinear horizontal diffusion coefficient [Eq. (54)], which proved to work sufficiently well. Nevertheless, it may be desirable to derive a more physically based extension of the scheme.

Future work will concentrate on testing the proposed nonlinear horizontal diffusion scheme in a mechanistic GCM with high spatial resolution in order to study gravity waves. Furthermore, we plan to test our extended nonlinear scheme in a comprehensive climate model with complex physical parameterizations in order to analyze its implications for climate sensitivity simulations.

Acknowledgments. The work was partly funded by the BMBF in the DEKLIM program. The important and helpful comments on the manuscript by William
Skamarok and two anonymous reviewers are gratefully acknowledged. We furthermore thank Kevin Hamilton, Michael Ponater, Robert Sausen, Charles McLandress, and Gerhard Schmitz for inspiring discussions.

\section{REFERENCES}

Alexeev, V. A., E. M. Volodin, and V. Ya. Galin, 1996: On the role of dissipation in the formation of atmospheric circulation. Izv. Atmos. Oceanic Phys., 32, 708-714.

Andrews, D. G., J. D. Mahlmann, and R. W. Sinclair, 1983: Eliassen-Palm diagnostics of wave-mean flow interaction in the GFDL "SKYHI" general circulation model. J. Atmos. Sci., 40, 2768-2784.

Barnes, J. R., and R. E. Young, 1992: Nonlinear baroclinic instability on the sphere: Multiple life cycles with surface drag and thermal damping. J. Atmos. Sci., 49, 861-878.

Becker, E., 2001: Symmetric stress tensor formulation of horizontal momentum diffusion in global models of atmospheric circulation. J. Atmos. Sci., 58, 269-282.

_ 2003a: Frictional heating in global climate models. Mon. Wea. Rev., 131, 508-520.

_ 2003b: Energetics of turbulent momentum diffusion and gravity wave breakdown in general circulation models of the atmosphere. Habilitation, Universität Rostock, 122 pp.

—_, and G. Schmitz, 2001: Interaction between extratropical stationary waves and the zonal mean circulation. J. Atmos. Sci., $\mathbf{5 8}, 462-480$.

_ , and D. C. Fritts, 2006: Enhanced gravity-wave activity and interhemispheric coupling during the MaCWAVE/MIDAS northern summer program 2002. Ann. Geophys., 24, 11751188.

Boville, B. A., 1991: Sensitivity of simulated climate to model resolution. J. Climate, 4, 469-485.

Burkhardt, U., and E. Becker, 2006: A consistent diffusiondissipation parameterization in the ECHAM climate model. Mon. Wea. Rev., 134, 1194-1204.

DKRZ, 1992: The ECHAM3 atmospheric general circulation model. Tech. Rep. 6, DKRZ, Hamburg, Germany, 184 pp.

Gordon, C. T., and W. F. Stern, 1982: A description of the GFDL global spectral model. Mon. Wea. Rev., 110, 625-644.

Grell, G. A., J. Dudhia, and D. R. Stauffer, 1995: A description of the fifth-generation Penn State/NCAR mesoscale model (MM5). NCAR Tech. Note NCAR/TN-398+STR, NCAR, Boulder CO, $122 \mathrm{pp}$.

Hamilton, K., R. J. Wilson, J. D. Mahlman, and L. J. Umscheid, 1995: Climatology of the SKYHI troposphere-stratospheremesosphere general circulation model. J. Atmos. Sci., 52, $5-43$.

,-- , and R. S. Hemler, 1999: Middle atmosphere simulated with high vertical and horizontal resolution versions of a GCM: Improvements in the cold pole bias and generation of a QBO-like oscillation in the Tropics. J. Atmos. Sci., 56, 3829-3846.

Holtslag, A. A. M., and B. A. Boville, 1993: Local versus nonlocal boundary-layer diffusion in a global climate model. J. Climate, 6, 1825-1842.

Kiehl, J. T., J. J. Hack, G. B. Bonan, B. A. Boville, B. P. Briegleb, D. L. Williamson, and P. J. Rasch, 1996: Description of the NCAR community climate model (CCM3). NCAR Tech. Note NCAR/TN-420+STR, NCAR, Boulder, CO, 152 pp.

Körnich, H., G. Schmitz, and E. Becker, 2003: Dependence of the annular mode in the troposphere and stratosphere on orog- 
raphy and land-sea heating contrasts. Geophys. Res. Lett., 30, 1265, doi:10.1029/2002GL016327.

Koshyk, J. N., and K. Hamilton, 2001: The horizontal kinetic energy spectrum and spectral budget simulated by a highresolution troposphere-stratosphere-mesosphere GCM. $J$. Atmos. Sci., 58, 329-348.

— , B. A. Boville, K. Hamilton, E. Manzini, and S. Shibata, 1999: Kinetic energy spectrum of horizontal motions in middle-atmosphere models. J. Geophys. Res., 104 (D22), 27 177-27 190.

Laursen, L., and E. Eliasen, 1989: On the effects of the damping mechanisms in an atmospheric general circulation model. Tellus, 41A, 385-400.

Lorenz, E. N., 1955: Available potential energy and the maintenance of the general circulation. Tellus, 7, 157-167.

- 1967: The Nature and Theory of the General Circulation of the Atmosphere. WMO Monogr., No. 218, WMO, 161 pp.

Oort, A. H., 1964: On estimates of the atmospheric energy cycle. Mon. Wea. Rev., 92, 483-493.

O'Sullivan, D., and T. J. Dunkerton, 1995: Generation of inertiagravity waves in a simulated life cycle of baroclinic instability. J. Atmos. Sci., 52, 3695-3716.

Roeckner, E., and Coauthors, 1992: Simulation of the present-day climate with the ECHAM model: Impact of model physics and resolution. Max-Planck-Institut für Meteorologie Rep. 93, Hamburg, Germany, 172 pp.

-, and Coauthors, 1996: The atmospheric general circulation model ECHAM-4: Model description and simulation of present-day climate. Max-Planck-Institut für Meteorologie Rep. 218, Hamburg, Germany, 90 pp.

Salmon, R., 1998: Geophysical Fluid Dynamics. Oxford University Press, $378 \mathrm{pp}$.

Simmons, A. J., and B. J. Hoskins, 1978: The life cycles of some nonlinear baroclinic waves. J. Atmos. Sci., 35, 414-432.

_- , and D. M. Burridge, 1981: An energy and angularmomentum conserving vertical finite-difference scheme and hybrid vertical coordinates. Mon. Wea. Rev., 109, 758-766.

Smagorinsky, J., 1963: General circulation experiments with the primitive equations. 1. The basic experiment. Mon. Wea. Rev., 91, 99-164.

- 1993: Some historical remarks on the use of nonlinear viscosities. Large Eddy Simulation of Complex Engineering and Geophysical Flows, B. Galperin and St. A. Orszag, Ed., Cambridge University Press, 3-36.

Smith, K. S., 2004: Comments on "The $k^{-3}$ and $k^{-5 / 3}$ energy spectrum of atmospheric turbulence: Quasigeostrophic two-level model simulation." J. Atmos. Sci., 61, 937-942.

Sommerfeld, A., 1978: Mechanik der Deformierbaren Medien. Verlag Harri Deutsch, 446 pp.

Thuburn, J., 1997: A PV-based shallow-water model on a hexagonal-icosahedral grid. Mon. Wea. Rev., 125, 2328-2347.

Tung, K. K., 2004: Reply. J. Atmos. Sci., 61, 943-948.

—, and W. W. Orlando, 2003: The $k^{-3}$ and $k^{-5 / 3}$ energy spectrum of atmospheric turbulence: Quasigeostrophic two-level model simulation. J. Atmos. Sci., 60, 824-835.

Wilks, D. S., 1995: Statistical Methods in the Atmospheric Sciences. Academic Press, 464 pp. 